\title{
The density maps of the HS47.5-22 field
}

\author{
S. N. Dodonov ${ }^{1}$ and A. Grokhovskaya ${ }^{1}$ \\ Special Astrophysical Observatory of the Russian Academy of Science, \\ Nizhnii Arkhyz, 369167, Russia
}

Received: July 31, 2019; Accepted: November 14, 2019

\begin{abstract}
We study the reconstruction of overdensity maps of galaxies as function of redshift in the range $0<\mathrm{z}<0.8$ using data from 1-m Schmidt Telescope of Byurakan Astrophysical Observatory (Armenia) in 16 medium band $(\sim 250 \AA)$ and four broad band (u,g,r,i) filters. The data used in this work homogeneously cover 2.39 sq. deg with accurate photometric redshifts, down to $\mathrm{R}<23 \mathrm{mag}(\mathrm{AB})$. We reconstructed the density contrast maps for the whole galaxy sample of the HS 47.5-22 ROSAT field in narrow slices for full range of redshifts. We select groups and clusters of galaxies with adaptive kernel based on density peaks which are larger than two times the mean density. The reconstructed overdensity field of galaxies consists of cluster-like structures outlining void-like regions for full redshift range $0 \leq \mathrm{z} \leq 0.8$. We detect known galaxy clusters in this field with software specially developed for this project. This gives us a possibility to study how star formation properties and galaxy morphology depend on the environments of the galaxies in this field.
\end{abstract}

Key words: methods - data analysis - galaxies - clusters

\section{Introduction}

Galaxy evolution and physical properties must be in strong correlation with their environment. There are a several dependencies of galaxy physical properties and the environment. The dependence "morphology - density of the environment" was discovered by Oemler (1974) and Dressler (1980). These authors stated that the early type galaxies are more often located in overdensity areas in the center of groups and clusters, and that the late type galaxies are preferentially found in the periphery of groups and clusters. More recent studies based on 2dFGRS (Two-degree-Field Galaxy Redshift Survey, Madgwick et al., 2003) and SDSS (Sloan Digital Sky Survey, Guo et al., 2013, 2014) have found that this correlation is maintained for the entire range of local densities up to the field galaxies.

For nearby galaxies the dependencies of $\mathrm{H}_{\alpha}$ equivalent width, $4000 \AA$ break (or the ratio between the average flux density in ergs s${ }^{-1} \mathrm{~cm}^{-2} \mathrm{~Hz}^{-1}$ between 4050 and $4250 \AA$ and that between 3750 and $3950 \AA$ Bruzual, 1983) and colors on the environment were found on scales of the order of $1 \mathrm{Mpc} \mathrm{h}^{-1}$ (Kauffmann et al., 2004). In the recent article of Cucciati et al. (2010) it was shown that more massive galaxies were formed in the most dense areas earlier than galaxies 
with a smaller mass and the evolution of less massive galaxies occurs under the influence of complex physical processes determined by their environment.

Galaxies located in areas of high density of groups and clusters of galaxies are formed and evolve differently than galaxies in the low density voids. One of the main methods for determination of galaxies belonging to a large-scale structure, in the optical and infrared ranges, is density contrast maps based on filtering algorithms (Lopes et al., 2004; Koester et al., 2007).

In this paper we describe the survey observations and the data reduction in Section 2. We review filtering algorithm with adaptive kernel, basic statistics and overdensity field reconstruction in Section 3. We discuss our results and make conclusion in Section 4. Throughout this paper, we assume a flat cosmology described with $\Omega_{m}=0.3, \Omega_{\Lambda}=0.7$ and $\mathrm{H}_{0}=70 \mathrm{~km} \mathrm{~s}^{-1}$.

\section{Observations}

We observed the HS47.5-22 ROSAT field (Fig. 1) defined by the medium deep ROSAT survey with center coordinates $09^{h} 50^{m} 00^{s}+47^{d} 35^{m} 00^{s}$ (Molthagen et al., 1997). The survey consists of 48 overlapping ROSAT pointings which were added to produce a final catalog containing $574 \mathrm{X}$-ray sources with broad band $(0.1-2.4 \mathrm{keV})$ count rates between $\sim 3 \times 10^{-3} \mathrm{cts} \mathrm{s}^{-1}$ and $\sim 0.2 \mathrm{cts} \mathrm{s}^{-1}$, in a field of view of $\sim 2.3 \mathrm{sq}$. deg. Molthagen et al. (1997) used an X-ray error circle of $2 \sigma+10$ arcsec in radius, with the positioning error box $\sigma$ for ROSAT objects taken from ROSAT observations.

Observations of the field HS47.5-22 were carried out with 1-m Schmidt Telescope of the Byurakan Observatory (Armenia) during several sets in February, March, April, and November of 2017, and in February and November of 2018. Telescope field of view with $4 k \times 4 k$ CCD was $58 \times 58$ arcmin, scale 0.868 arcsec pixel $^{-1}$. To get data for near all pointings of HS47.5-22 ROSAT field we observed four positions with 10 arcmin overlaps. From these observations we created the mosaic with total area of 2.386 sq. degree (Dodonov et al., 2017).

Each position was observed in four broad band filters $(u, g, r$ and $i S D S S)$ and in 16 medium band $(F W H M=250 \AA$, with FWHM defined as full width at half maximum) filters with homogeneous covering optical spectral range from $4000 \AA$ till $8000 \AA$ (see Tab. 1 and Fig. 2). In each filter we obtained from 12 to 25 individual images with 5-6 arcmin shifts between each one. Total exposure times in filters were varied from $60 \mathrm{~min}$ to $120 \mathrm{~min}$ depending from the spectral sensitivity of the CCD. Long term objects variability was controlled by the observations in $r-S D S S$ filter in each set of observations.

Photometry of the objects obtained using SExtractor (Bertin \& Arnouts, 1996) in dual image mode. Base image were created from the sum of deep $\left(\sim 25^{m}\right)$ images obtained in $g, r$ and $i$ filters. Before the photometry all images were convolved to common seeing quality and transformed to common coordi- 


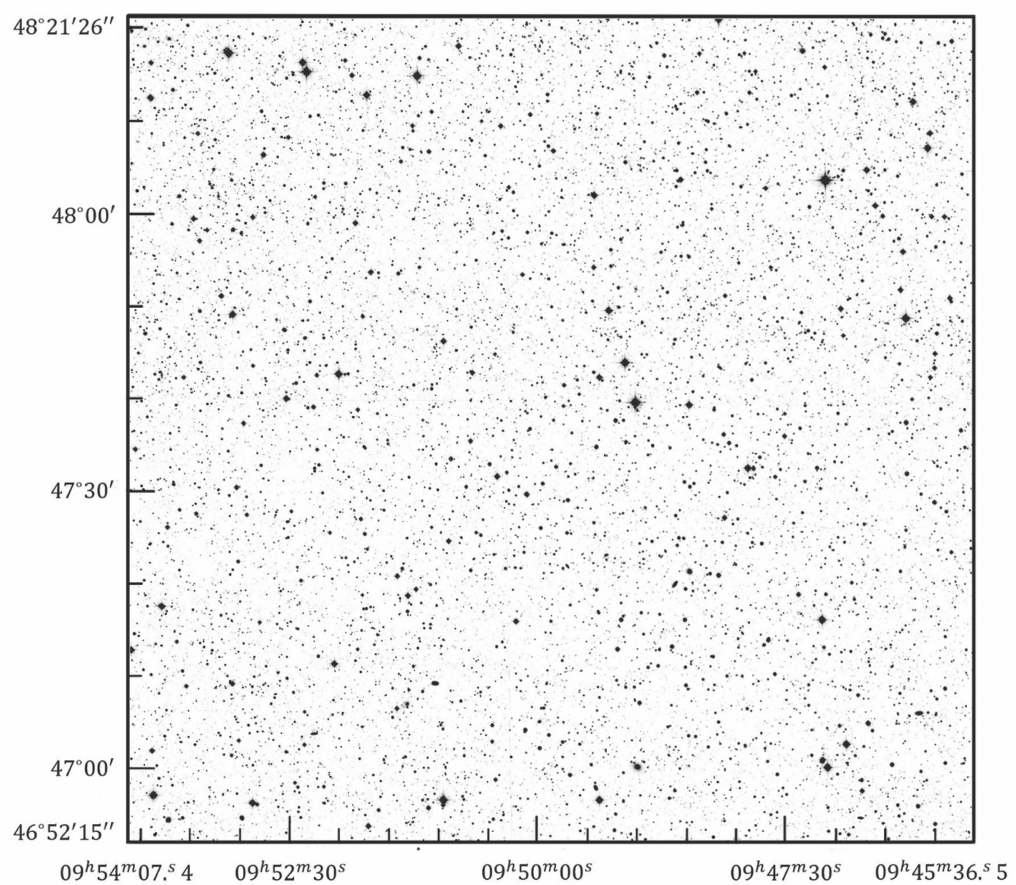

Figure 1. The composite optical image in $\mathrm{g}, \mathrm{r}$ and i filters of the HS47.5-22 field is about 2.39 sq. degree with center coordinates $09^{h} 50^{m} 00^{s}+47^{d} 35^{m} 00^{s}$. The $574 \mathrm{X}$-ray sources up to level $3.5 \cdot 10^{-14} \mathrm{erg} \mathrm{cm}^{-2} \mathrm{~s}^{-1}$ were detected by the ROSAT X-ray satellite (Molthagen et al., 1997).

nates system. We got photometry in $1.45 \times F W H M$ apertures and corrected received fluxes for light loss using light curve obtained from bright stars, and using MAG_AUTO SExtractor photometry we receive Kron-like fluxes for all objects in the field. Photometric calibration was developed using spectral and photometrical data from SDSS survey for the objects detected in the field. By using field objects as a standard stars within each exposure, we were independent from photometrical conditions for imaging.

Galaxies sample is extracted from the full catalog of objects (near 100000 objects) using following criteria:

- Objects brighter then $R_{A B}=23$ mag ( $\mathrm{AB}$ magnitude in $\mathrm{R}$ filter)

- Extended index $<0.8$ (Bertin \& Arnouts, 1996) for the objects with $R_{A B}<$ $21 \mathrm{mag}$, extended index $<0.9$ for the objects with $21 \mathrm{mag}<R_{A B}<22$ mag and extended index $<0.96$ for the objects with $R_{A B}<23$ mag

- Index of contamination $\leq 2$ (Bertin \& Arnouts, 1996) 
Table 1. $1 \mathrm{~m}$ Schmidt Telescope Filter set. Effective Wavelength, FWHM, Limiting Magnitude measured at $\mathrm{F} / 2$.

\begin{tabular}{cccc}
\hline \hline Filter & $\lambda_{\text {cen }}, \AA$ & $F W H M(\AA)$ & $m_{\lim , 5 \sigma}$ \\
\hline u_SDSS & 3578 & 338 & 24.23 \\
g_SDSS & 4797 & 860 & 25.22 \\
r_SDSS & 6227 & 770 & 24.97 \\
i_SDSS & 7624 & 857 & 24.15 \\
MB_400 & 3978 & 250 & 24.37 \\
MB_425 & 4246 & 250 & 24.31 \\
MB_450 & 4492 & 250 & 24.20 \\
MB_475 & 4745 & 250 & 24.31 \\
MB_500 & 4978 & 250 & 24.30 \\
MB_525 & 5234 & 250 & 24.37 \\
MB_550 & 5496 & 250 & 23.86 \\
MB_575 & 5746 & 250 & 24.29 \\
MB_600 & 5959 & 250 & 23.89 \\
MB_625 & 6234 & 250 & 23.51 \\
MB_650 & 6499 & 250 & 23.41 \\
MB_675 & 6745 & 250 & 23.78 \\
MB_700 & 7002 & 250 & 23.47 \\
MB_725 & 7253 & 250 & 23.20 \\
MB_750 & 7519 & 250 & 23.07 \\
MB_775 & 7758 & 250 & 22.97 \\
\hline
\end{tabular}

The sample of objects which follow the first two criteria includes 40194 objects and after applying the third one - we have 32637 objects with clean photometry. Due to the contamination we lose $8.12 \%$ of the objects.

We check sample completeness using comparison of galaxies number-counts in $g, r$ and $i S D S S$ filters from our sample with already published data in Fig. 3. The galaxies sample completeness up to $R_{A B}=23$ mag with no color selection effects in all optical range.

Photometric measurements from 17 filters $(u-S D S S$ plus 16 medium band filters) provide low resolution spectra Fig. 4 for each object which are analyzed by a statistical technique for classification and redshift estimation based on spectral template matching. We used for these galaxies spectra templates library from Dodonov \& Chilingarian (2008), and a set of programs ZEBRA (Zurich's Extragalactic Bayesian Redshift Analyzer, Feldmann et al., 2006).

The obtained redshift accuracy $\sigma_{z}<0.01$ and the fraction of catastrophic outliers is $\left(\Delta z /(1+z)>5 . * \sigma_{z}\right) \sim 4.0 \%$ (in Fig. 5). Accuracy $\sigma_{z}$ changes from 0.01 in magnitude range $r-S D S S=16 \mathrm{mag}-21 \mathrm{mag}$ till 0.03 in magnitude range $r-S D S S=21 \mathrm{mag}-23 \mathrm{mag}$. 


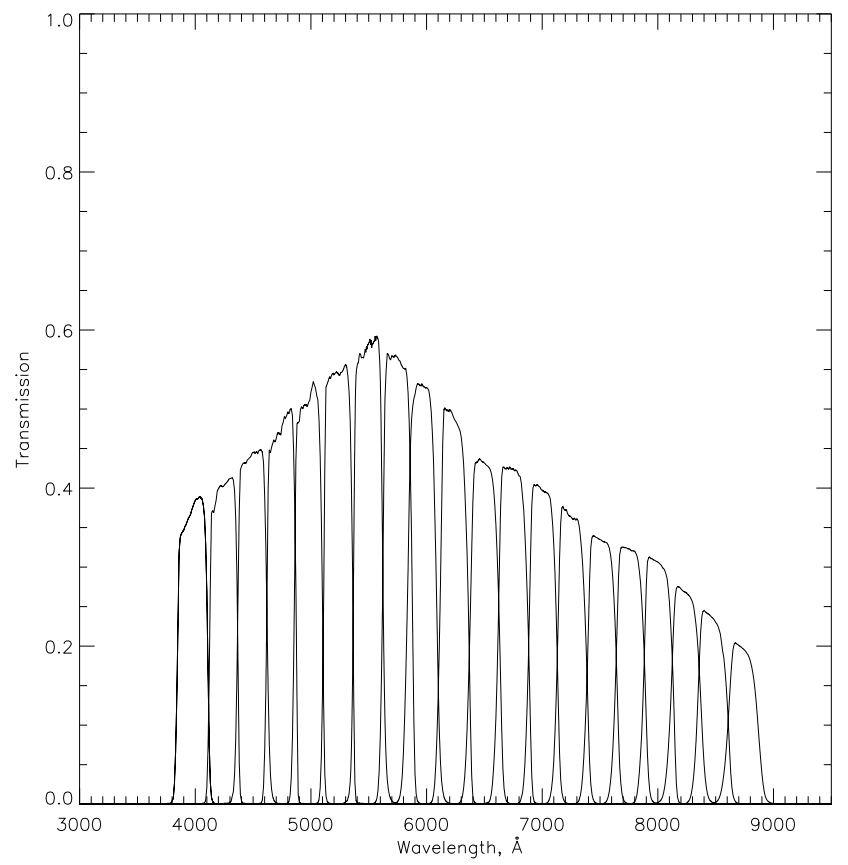

Figure 2. 1m Schmidt Telescope Filter set. Filter transmission measured at F/2. CCD spectral response included.

\section{Data analysis}

\subsection{Filtering algorithm with adaptive kernel}

In this work we use the algorithm with adaptive kernel to reconstruct the density contrast field. In this method we calculate the density value in the vicinity of each galaxy:

$$
\delta_{i}=\frac{s}{\frac{4}{3} \pi R^{3}},
$$

where $R$ is three dimensional distance from the galaxy to its N-th nearest neighbor and $s$ is number of nearest neighbor galaxy. Next we divide the light cone into thin redshift slices with $\Delta z=0.056 \cdot(1+z)$ and $25 \%$ overlap from each side of slice. The width of slices is based on $1 \sigma=0.028$ photometric redshift error for all type galaxies. For each slice the mean density is estimated as

$$
\bar{\delta}=\frac{1}{n} \sum_{i=1}^{n} \delta_{i},
$$

where $n$ is the total number of galaxies in the each slice. 

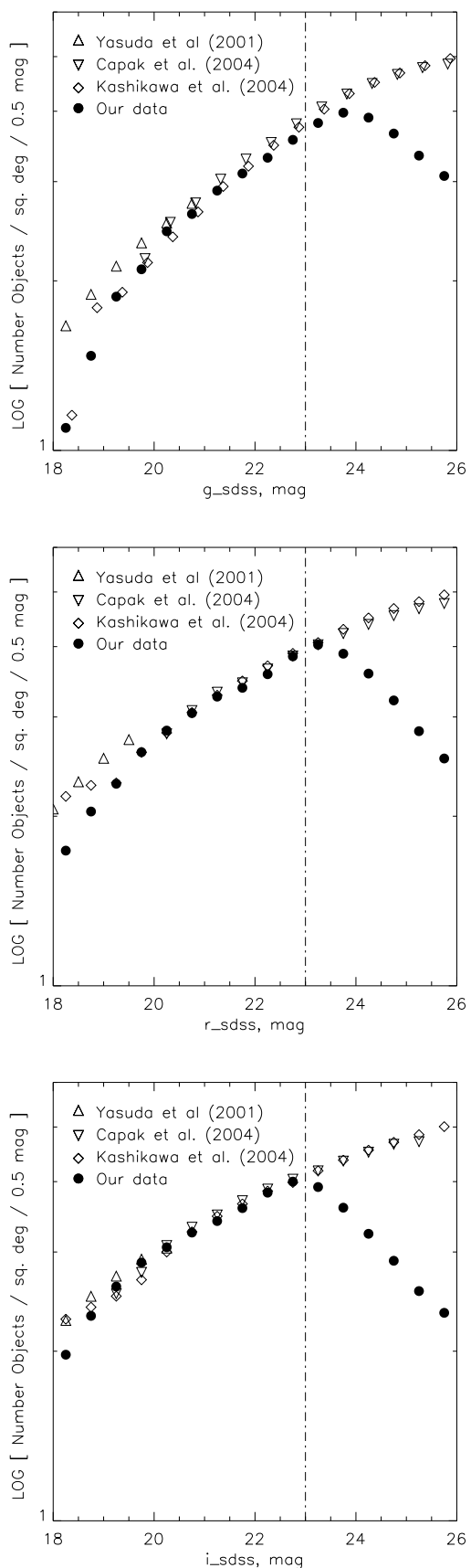

Figure 3. Galaxy sample completeness in $g, r$ and $i S D S S$ filters. 

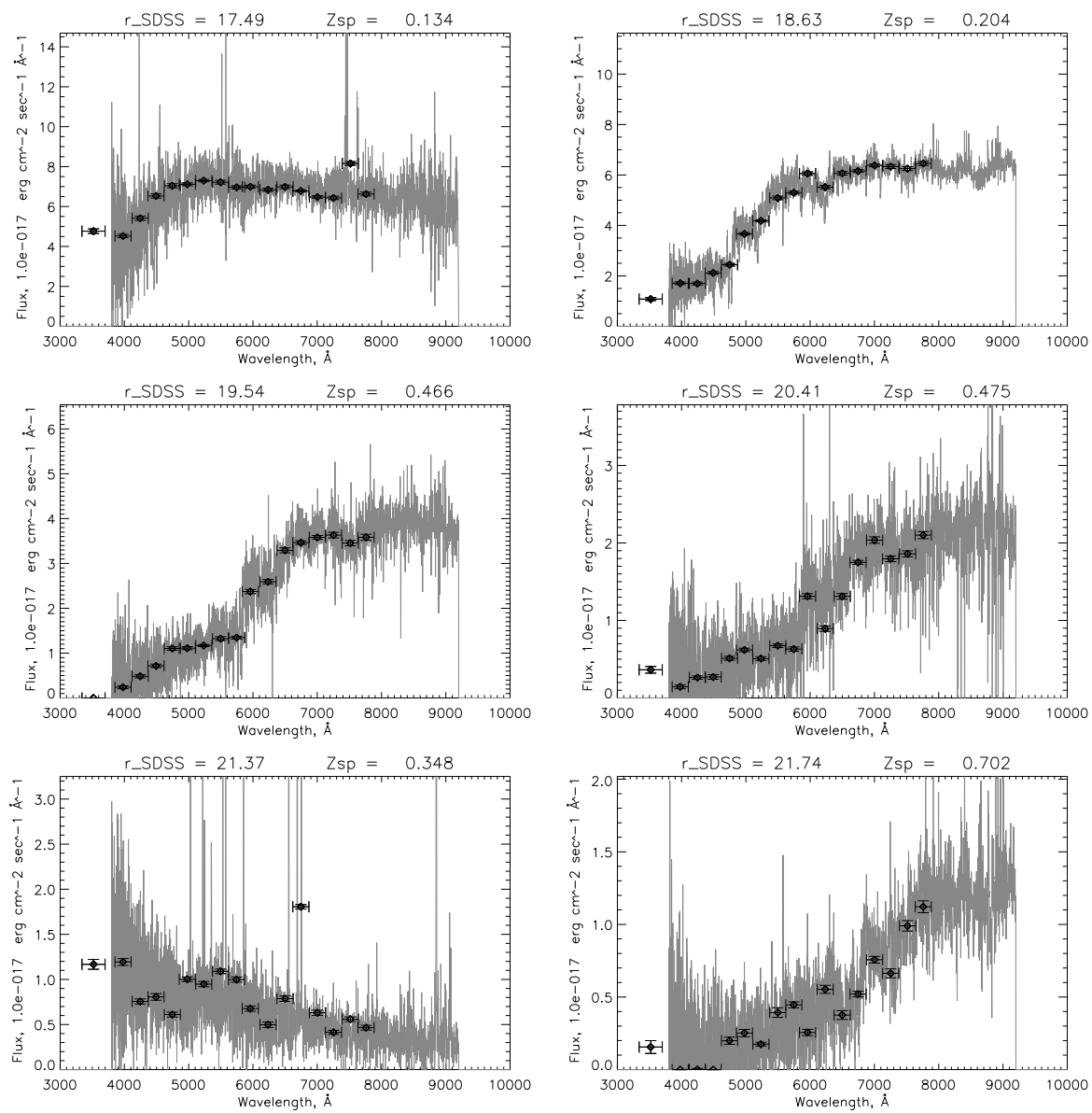

Figure 4. Comparison of the Spectral Energy Distribution (SED) obtained from the photometric data with 1-meter Schmidt telescope (points with bars) with the SDSS spectra (solid gray line). Points in the SED are scaled to 3 arcsec to match the SDSS fiber diameter. Horizontal bars corresponds to filter width and vertical bars to $1 \sigma$ flux error.

The density contrast $\sigma_{i}$ for each galaxy position is calculated as

$$
\sigma_{i}+1=\frac{\left(\delta_{i}-\bar{\delta}\right)}{\bar{\delta}}+1
$$

The candidate to clusters and group of galaxies are detected as density peaks which are larger than two times the average density and the candidate to voids 

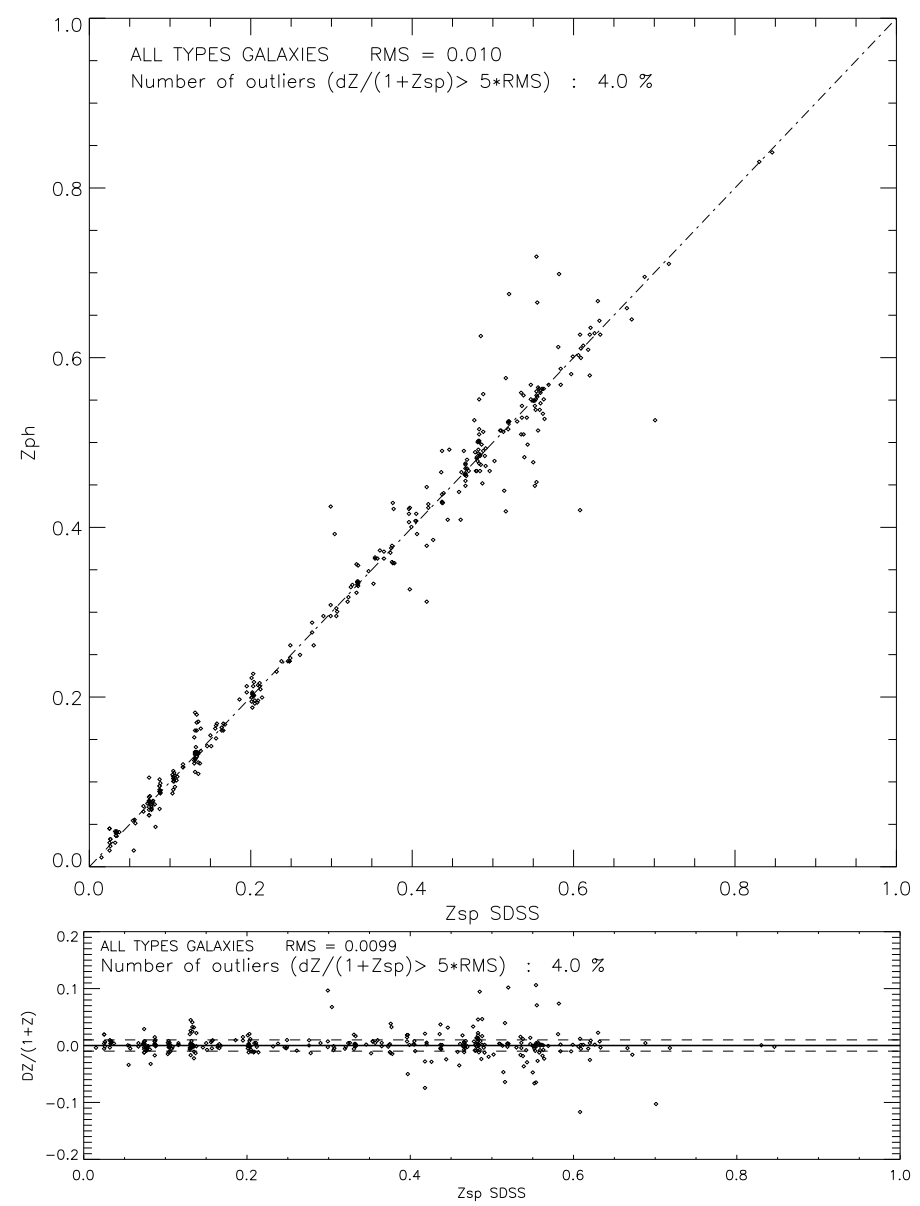

Figure 5. Comparison between photometric redshifts $z_{p h}$ obtained with ZEBRA in Maximum Likelihood mode with SDSS spectroscopic redshifts $z_{s p}$ along with error distribution $\Delta z /(1+z)$ for all galaxies with known spectroscopic redshifts

are detected as density cavity smaller than ten times the average density in each redshift slice.

\subsection{Basic statistics}

For testing filtering algorithm with adaptive kernel we use galaxy mock-catalog MICECAT v2 (Carretero et al., 2017). It is a fake or simulated galaxy catalogue which resembles a genuine galaxy redshift survey catalogue, but is built from a cosmological simulation. Mock catalog is useful for cluster-determination 
algorithms because it has information about dark halo id. Thus we can estimate statistical parameters of detecting clusters sample over mock sample. Obviously, it is impossible to obtain a perfect match between structures of the model catalog and the recovered groups and clusters. Moreover completeness and purity of reconstructed structures tend to be mutually exclusive. In the process of determining the elements of a large-scale structure, it is possible to obtain the following cases: over-merging (several real groups combined to one reconstructed group), fragmentation (one real group divided to a several reconstructed groups), spurious group (reconstructed group with no one real group matching), undetected group (real group is missing in reconstructed catalog).

Basic statistic term are: I) the completeness, which is a measure of the fraction of real groups with $\mathrm{N}$ or more members that are successfully recovered in the reconstructed group catalogue, II) the purity, which is a measure of the fraction of reconstructed groups with $\mathrm{N}$ or more members that belong to real groups, III) "galaxy success rate" which is the fraction of galaxies belonging to real groups of special richness that have ended up in any reconstructed group, and IV) "interloper fraction" is the fraction of galaxies belonging to reconstructed groups of special richness which are field galaxies (Knobel et al. (2009), see also Grokhovskaya \& Dodonov (2019)).
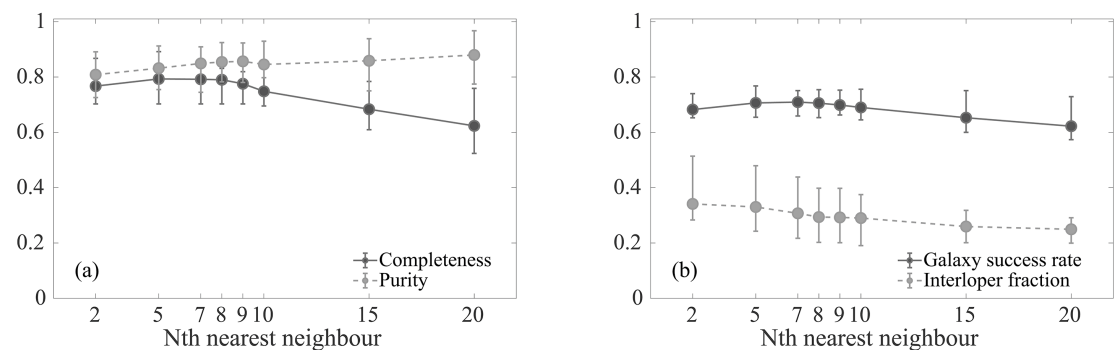

Figure 6. Basic static parameters of the sample of groups, clusters and galaxies obtained by filtering algorithm with adaptive kernel. On the left the figures show completeness (solid line) and purity (dashed line) of the sample, on the right - the percentage of successful detection of galaxies from clusters of the model catalog (solid line) and the percentage of galaxies defined automatically as galaxies of clusters, but being field galaxies in the model catalog (dotted line). The bars correspond to the standard deviation for 10 samples of galaxies of the MICE simulation.

We use 10 mock samples with the same physical properties like observation data ( 2 sq. degree field, $\mathrm{z} \leq 0.8$ and $R_{A B} \leq 23 \mathrm{mag}$ ) for estimation of all basic statistical parameters for detected clusters by filtering algorithm with adaptive kernel (Fig. 6). For this method we can variate size of aperture as number of nearest neighbor for calculation and compare statistics for choosing best size 
of aperture. The 8 nearest neighbours is optimal variant of completeness and purity.

\subsection{The HS47.5-22 overdensity field}

We reconstructed 3D overdensity maps for observational data of the HS 47.5-22 field. We obtained a broad range of reliably reconstructed local overdensities of the sample of galaxies by using filtering algorithm with variable aperture. The size of aperture was defined by the distance to the 8th nearest neighbour from basic statistics. Based on the tests on the mock catalogue, 8 is the optimal number of neighbours which can be used to reliably reconstruct density at all redshifts. An increase in the size of the adaptive aperture (e.g. 10, 15 or 20) leads to a smoothing of the peaks on the contrast maps of the density of galaxies and the underdetermination of large-scale structures. Decrease in the size of the adaptive aperture (e.g. 2, 5 or 7 ) leads to decrease of the purity of the reconstructed cluster sample.

Figure 7 shows isosurfaces for a density contrast which are larger than two times the average density (grey surfaces) and clusters with spectral redshifts from WHL (by the name of the authors of the catalog Wen, Han and Liu) catalog (black triangles) which was completed by Wen \& Han (2015) from spectroscopic and photometric data of the SDSS.

\section{Conclusion}

We used observational data from 1-m Schmidt Telescope of the Byurakan Observatory (Armenia) to reconstruct the overdensity maps in the HS47.5-22 ROSAT field. We explored the photometric properties of the sample of 36447 galaxies in the field and obtained spectral types and photometric redshifts for all objects. An accuracy of redshift allows one to determine whether a galaxy belongs to a cluster or group.

We applied the filtering algorithm with adaptive aperture for the reconstruction. This algorithm have been tested on the MICECAT v2 mock catalog. We estimated basic statistical parameters (completeness, purity, galaxy success rate and interloper fraction) for reconstructed clusters from mock catalog. Evaluation of the main statistical parameters allowed us to choose the optimal aperture size for further work with observational data.

We obtain a broad band of large scale structures up to $z=0.8$ for observational data. Also we found the most part of clusters from the WHL cluster catalog with spectroscopic redshift in our field.

Obtained results allow us to begin a study of the connection between star formation rate in galaxies and their position in the large scale distribution. 

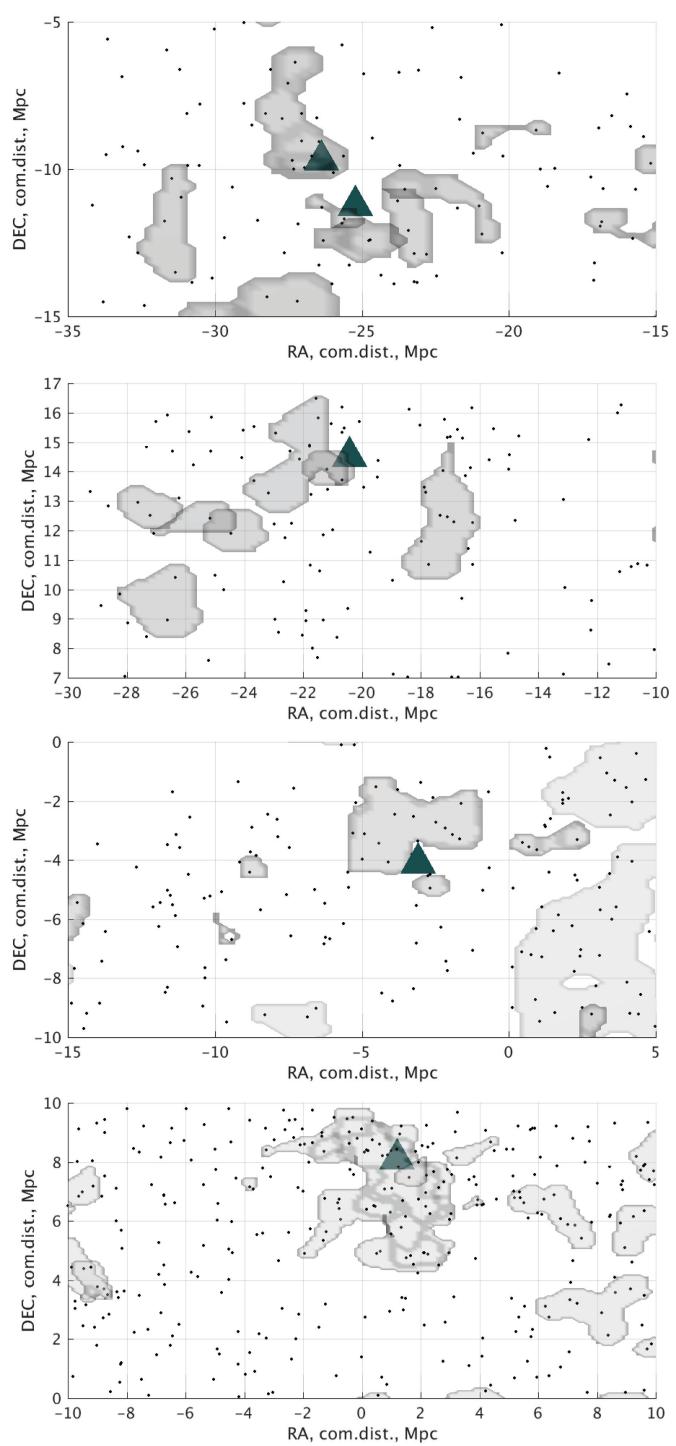

Figure 7. Overdensity maps (grey surfaces are isosurfaces with a density contrast two times above the mean density mean density) of narrow slices HS 47.5-22 field with WHL clusters (black triangles, from top to bottom): 1. WHL J094645.7 + 471107 and WHL J094637.9 +471440 2. WHL J094657.3 +481526 3. WHL J094913.0 +472248 4. WHL J095027.9 +481435. Axis are shown in comoving distance (x-axis is distance from the survey centre in right ascension, $\mathrm{y}$-axis is distance from the survey centre in declination). 
Acknowledgements. This work has made use of CosmoHub. CosmoHub has been developed by the Port d'Informacio Cientifica (PIC), maintained through a collaboration of the Institut de Fisica d'Altes Energies (IFAE) and the Centro de Investigaciones Energeticas, Medioambientales y Tecnologicas (CIEMAT), and was partially funded by the "Plan Estatal de Investigacion Cientifica y Tecnica y de Innovacion" program of the Spanish government.

The methodological part of the work was supported by the Russian Science Foundation under grant no. 17-12-01335 (observation, development of methods for processing and calibrating data).

The data analysis and the study of the large-scale structure were performed as part of the government contract of the SAO RAS approved by the Ministry of Science and Higher Education of the Russian Federation.

\section{References}

Bertin, E. \& Arnouts, S., SExtractor: Software for source extraction. 1996, Astronomy and Astrophysics Supplement, 117, 393, DOI: 10.1051/aas:1996164

Bruzual, A., G., Spectral evolution of galaxies. I. Early-type systems. 1983, Astrophysical Journal, 273, 105, DOI: 10.1086/161352

Carretero, J. et al., CosmoHub and SciPIC: Massive cosmological data analysis, distribution and generation using a Big Data platform. 2017, PoS, EPS-HEP2017, 488, DOI: $10.22323 / 1.314 .0488$

Cucciati, O., Iovino, A., Kovac, K., et al., The zCOSMOS 10k-sample: the role of galaxy stellar mass in the colour-density relation up to z 1. 2010, Astronomy and Astrophysics, 524, 18, DOI: 10.1051/0004-6361/200912585

Dodonov, S. N. \& Chilingarian, I. V., Library of reference spectral energy distributions for galaxies for the classification and determination of photometric redshifts of objects. 2008, Astrophysical Bulletin, 63, 1, DOI: 10.1007/s11755-008-1001-y

Dodonov, S. N., Kotov, S. S., Movsesyan, T. A., \& Gevorkyan, M., One-meter Schmidt telescope of the Byurakan Astrophysical Observatory: New capabilities. 2017, Astrophysical Bulletin, 72, 473, DOI: 10.1134/S1990341317040113

Dressler, A., Galaxy morphology in rich clusters - Implications for the formation and evolution of galaxies. 1980, The Astrophysical Journal, 236, 351, DOI: $10.1086 / 157753$

Feldmann, R., Carollo, C. M., Porciani, C., et al., The Zurich Extragalactic Bayesian Redshift Analyzer and its first application: COSMOS. 2006, Monthly Notices of the Royal Astronomical Society, 372, 565, DOI: 10.1111/j.1365-2966.2006.10930.x

Grokhovskaya, A. A. \& Dodonov, S. N. 2019, in prep.

Guo, H., Zehavi, I., Zheng, Z., et al., The Clustering of Galaxies in the SDSS-III Baryon Oscillation Spectroscopic Survey: Luminosity and Color Dependence and Redshift Evolution. 2013, The Astrophysical Journal, 767, 1, DOI: 10.1088/0004637X/767/2/122

Guo, H., Zheng, Z., Zehavi, I., et al., The clustering of galaxies in the SDSS-III Baryon Oscillation Spectroscopic Survey: modelling of the luminosity and colour depen- 
dence in the Data Release 10. 2014, Mon. Not. R. Astron. Soc., 441, 2398, DOI: $10.1093 / \mathrm{mnras} / \mathrm{stu} 763$

Kauffmann, G., White, S. D. M., Heckman, T. M., et al., The environmental dependence of the relations between stellar mass, structure, star formation and nuclear activity in galaxies. 2004, Mon. Not. R. Astron. Soc., 353, 713, DOI: 10.1111/j.13652966.2004.08117.x

Knobel, C., Lilly, S. J., Iovino, A., et al., An Optical Group Catalog to z $=1$ from the zCOSMOS $10 \mathrm{k}$ Sample. 2009, The Astrophysical Journal, 697, 1842, DOI: 10.1088/0004-637X/697/2/1842

Koester, B. P., McKay, T. A., Annis, J., et al., MaxBCG: A Red-Sequence Galaxy Cluster Finder. 2007, The Astrophysical Journal, 660, 221, DOI: 10.1086/512092

Lopes, P. A. A., de Carvalho, R. R., Gal, R. R., et al., The Northern Sky Optical Cluster Survey. IV. An Intermediate-Redshift Galaxy Cluster Catalog and the Comparison of Two Detection Algorithms. 2004, The Astrophysical Journal, 128, 1017, DOI: $10.1086 / 423038$

Madgwick, D. S.and Hawkins, E., Lahav, O., Maddox, S., et al., The 2dF Galaxy Redshift Survey: galaxy clustering per spectral type. 2003, Mon. Not. R. Astron. Soc., 344, 847, DOI: 10.1046/j.1365-8711.2003.06861.x

Molthagen, K., Wendker, H. J., \& Briel, U. G., Multiwavelength observations of the field HS 47.5/22 in Ursa Major. I. The X-ray catalogue of a medium deep ROSAT survey. 1997, Astronomy \& Astrophysics Supplement series, 126, 509, DOI: 10.1051/aas:1997391

Oemler, A. 1974, The systematic properties of clusters of galaxies, PhD dissertation, California Institute of Technology

Wen, Z. L. \& Han, J. L., Calibration of the Optical Mass Proxy for Clusters of Galaxies and an Update of the WHL12 Cluster Catalog. 2015, The Astrophysical Journal, 807, 11pp, DOI: 10.1088/0004-637X/807/2/178 